\title{
Successful treatment of arrhythmia with $\beta$-blocker and Flecainide combination in pregnant patients with Andersen-Tawil syndrome
}

\author{
Pongprueth Rujirachun ${ }^{1}$, Apichaya Junyavoraluk ${ }^{1}$, Manop Pithukpakorn ${ }^{1}$, Bhoom \\ Suktitipat ${ }^{1}$, and Arjbordin Winijkul ${ }^{1}$ \\ ${ }^{1}$ Mahidol University Faculty of Medicine Siriraj Hospital
}

June 9, 2020

\begin{abstract}
Andersen-Tawil syndrome (ATS) is a rare inherited ventricular arrhythmia (VA). Here, we reported two KCNJ2-associated ATS patients who got pregnant and underwent vaginal birth safely. Both individuals had VA, micrognathia without periodic paralysis. $\beta$-blocker plus flecainide could be an effective treatment combination when monotherapy failed to control arrhythmia.
\end{abstract}

\section{Introduction}

Andersen-Tawil syndrome (ATS) is a rare autosomal dominant channelopathy characterized by clinical triad of ventricular arrhythmia (VA), periodic paralysis and distinctive dysmorphic features.

Due to the rarity of this condition, less is known about physiologic effect of pregnancy to ATS and arrhythmia. There is no established guideline for prenatal or postpartum treatment and prevention of arrhythmia in ATS, thus the clinical management is challenging ${ }^{1}$. Here we reported two pregnant ATS patients whose VA could be controlled by antiarrhythmic drug combination.

\section{Case report}

\section{Case \#1}

A 32-year-old Thai woman (Figure 1, ATS-003) came for a medical advice on pregnancy planning in 2014. She had a history of abnormal heart rhythm found at general check-up since childhood. She was asymptomatic and grew up normally. The patient had one sister (ATS-002) and three brothers. Her mother (ATS-001) and sister experienced a similar history of abnormal heart rhythm. Both of them were also asymptomatic and uneventful. All of her brothers were not affected. There was no history of sudden unexpected death in the family. She had another post-marital health check-up and multiform premature ventricular contractions (PVC) with a short run of non-sustained polymorphic ventricular tachycardia (PMVT) was uncovered by electrocardiography (ECG). (Figure 2A). Further cardiac investigation by magnetic resonance imaging together with transthoracic echocardiography (TTE) showed left ventricular ejection fraction (LVEF) $25.1 \%$, mild mitral valve regurgitation, mild tricuspid regurgitation, and no subendocardial scar. Only micrognathia was noted during physical examination without other dysmorphic features. Clinical diagnosis of Andersen-Tawil syndrome was suspected.

Genetic testing identified heterozygous likely pathogenic variant in KCNJ2 c.557C > G (p.Pro186Arg) in all affected members. (Figure 1). Treatment plan had been discussed between healthcare team and the patient. Catheter ablation was declined therefore oral Metoprolol was given. The medication dose was gradually increased to $100 \mathrm{mg}$ daily with no significant change of arrhythmias by periodic ECG monitoring. The patient decided to discontinue Metoprolol after she became pregnant. 
Her pregnancy was uneventful and cardiac evaluation was done regularly. The ventricular ectopy became less frequent (Figure 2B). TTE at gestational age of 24 weeks showed fair left ventricular contraction with left ventricular ejection fraction (LVEF) at 45-50\%. The patient was admitted to the hospital due to labor pain at 38 weeks gestation. Continuous cardiac and fetal monitoring was instituted. Maternal ventricular ectopy was infrequent. Emergency cesarean delivery was done under spinal anesthesia due to irregular fetal heartbeats. There was no intrapartum and immediate postpartum complication. The patient was then transferred to cardiac care unit (CCU) for postoperative observation.

During CCU stay, she developed persistent PMVT (Figure 2C). Metoprolol was resumed and $100 \mathrm{mg}$ of Flecainide daily was added to successfully suppress the arrhythmia. The patient continued to do well until now at 5 months postpartum with occasional ectopic rhythm without ventricular tachycardia (VT) (Figure 2D).

\section{Case \#2}

The 33 years old woman (ATS-002), sister of case \#1 (ATS-003), was also invited to our hospital for ATS evaluation. She was asymptomatic. Frequent bidirectional VT was noted on ECG (Figure 3A). TTE revealed fair left ventricular systolic function with LVEF at $52 \%$. Metoprolol was administered and gradually increased to $100 \mathrm{mg}$ daily but her VA was not significantly reduced. One hundred $\mathrm{mg}$ of Flecainide per day was then added and cardiac monitoring showed ventricular ectopy becoming less frequent without VT (Figure 3B). Four months after treatment combination, 24-hours Holter monitoring showed sinus rhythm with prominent U-wave and occasional ventricular ectopy, and good biventricular systolic function with trivial mitral valve regurgitation was observed by TTE.

Similar to her sister, the patient decided to underwent her pregnancy without medication after extensive discussion with healthcare team. Her pregnancy was uneventful and she delivered her child successfully. VT was noticed during postpartum monitoring, therefore, Flecainide and Metoprolol combination was resumed. Cardiac arrhythmia remained suppressed at 3 months follow-up visit (Figure 3C).

\section{Discussion}

Majority of ATS individuals are caused by heterozygous mutations in KCNJ2 which encodes for the $\alpha$ subunit of Kir2.1, an inward rectifier potassium channel responsible for late phase of cardiac repolarization. Classic ECG abnormalities include QTc prolongation and prominent U-wave. Various types of VA, including bidirectional VT and PMVT, are prevalent and could be identified in more than $80 \%$ of the patients, though sudden cardiac death does not commonly occur ${ }^{2}$. Current management strategy of ATS is mainly focused on suppression of ventricular ectopy to restore left ventricular function and prevent tachycardia-induced cardiomyopathy ${ }^{3}$.

These two KCNJ2 [c.557C>G (p.Pro186Arg)]-associated ATS patients who got pregnant and underwent vaginal birth safely without any medications were first reported. Both individuals had VA, micrognathia without periodic paralysis. $\beta$-blocker plus Flecainide were shown to be effective when $\beta$-blocker alone could not suppress their arrhythmias.

Though the mechanism remains unknown, physiologic changes associated with pregnancy could have significant impact on cardiac conduction in patients with ATS. QTc interval is hormonally regulated and increased estradiol is associated with shorter QTc interval via enhanced membrane trafficking of $K C N H 2{ }^{4}$. One reported case also showed marked reduction of ventricular ectopy during pregnancy ${ }^{1}$.

$\beta$-blockers are considered mainstay therapy for inherited long QT syndromes, though $\beta$-blocker-resistant cases do occur ${ }^{5}$. Flecainide is a fast-inward sodium channel blocker with pleiotropic effects to reduce the oscillatory potentials. It can also terminate VT by modulating calcium dynamic via inhibiting reverse-mode sodium-calcium exchanger or ryanodine receptor 2 and directly increasing Kir2.1 current with no significant impact on left ventricular function ${ }^{6}$. The medication was shown to be effective on reducing VA burden in multiple ATS patients ${ }^{2,3,7-11}$ (Table 1). The synergistic effect of $\beta$-blockers and Flecainide might favor a combination treatment in ATS. 
In concordance with previously reported cases, the combination of Flecainide and $\beta$-blocker was useful in patients with ATS when $\beta$-blocker monotherapy failed to control cardiac arrhythmias.

\section{Conclusion}

We reported 2 ATS patients who underwent an uneventful pregnancy and childbirth and had effective control of ventricular arrhythmias with combination treatment of $\beta$-blocker and Flecainide.

\section{Acknowledgements}

We wish to thank our patients for their cooperation and contribution to our study. We thank all physicians and health professionals for the patient's clinical care. This work was supported by Siriraj Research Development Grant (R015734003) to BS and Siriraj Chalermphrakiat Grant to MP, BS and AW.

\section{References}

1. Subbiah RN, Gula LJ, Skanes AC, et al. Andersen-Tawil syndrome: Management challenges during pregnancy, labor, and delivery. J Cardiovasc Electrophysiol . 2008; 19(9):987-9

2. Fox DJ, Klein GJ, Hahn A, et al. Reduction of complex ventricular ectopy and improvement in exercise capacity with flecainide therapy in Andersen-Tawil syndrome. Europace . 2008;10(8):1006-8.

3. Pellizzón OA, Kalaizich L, Ptácek LJ, et al. Flecainide Suppresses Bidirectional Ventricular Tachycardia and Reverses Tachycardia-Induced Cardiomyopathy in Andersen-Tawil Syndrome. J Cardiovasc Electrophysiol. 2007;19(1):95-7.

4. Anneken L, Baumann S, Vigneault P, et al. Estradiol regulates human QT-interval: Acceleration of cardiac repolarization by enhanced KCNH2 membrane trafficking. Eur Heart J . 2016;37(7):640-50.

5. Moss AJ, Zareba W, Hall WJ, et al. Effectiveness and limitations of $\beta$-blocker therapy in congenital longQT syndrome. Circulation . 2000;101(6):616-23.

6. Brembilla-perrot B, Amor M, Auque F, et al. Effect of flecainide on left ventricular ejection fraction. Eur Heart J.1987;8(7):754-61.

7. Van Ert HA, McCune EC, Orland KM, et al. Flecainide treats a novel KCNJ2 mutation associated with Andersen-Tawil syndrome. Hear Case Reports . 2017;3(2):151-154.

8. Hayashi H, Kawaguchi T, Horie M. Effect of Flecainide on T-Wave Alternans in Andersen-Tawil Syndrome. Ann Noninvasive Electrocardiol . 2014;19(4):383-6.

9. Bökenkamp R, Wilde AA, Schalij MJ, et al. Flecainide for recurrent malignant ventricular arrhythmias in two siblings with Andersen-Tawil syndrome. Hear Rhythm . 2007;4(4):508-11.

10. Janson CM, Poelzing S, Shah MJ. Combined inhibition of Na+ and $\mathrm{Ca} 2+$ channels: A novel paradigm for the treatment of incessant ventricular arrhythmias in Andersen-Tawil syndrome. Hear Rhythm . 2014;11(2):318-20.

11. Fernández M, Marín MDR, Fernández-Armenta J, et al. Response to flecainide test in Andersen-Tawil syndrome with incessant ventricular tachycardia. PACE - Pacing Clin Electrophysiol . 2018;41(4):429-432.

\section{Figure legend}

Fig. 1 Family pedigree. The proband (ATS-003) is indicated by the arrow. Family members with ATS are illustrated as a red color (ATS-001 and ATS-002). Unaffected family members are illustrated as open symbols. A circle represents a female and a square represents a male.

Fig. 2 Twelve-lead ECG at A. First visit, B. Antepartum period, C. Postpartum period, D. Postpartum period with flecainide 
Fig. 3 Twelve-lead ECG demonstrating A. Incessant bidirectional VT, B. Sinus rhythm with prominent U wave and occasional PVCs, C. Normal sinus rhythm
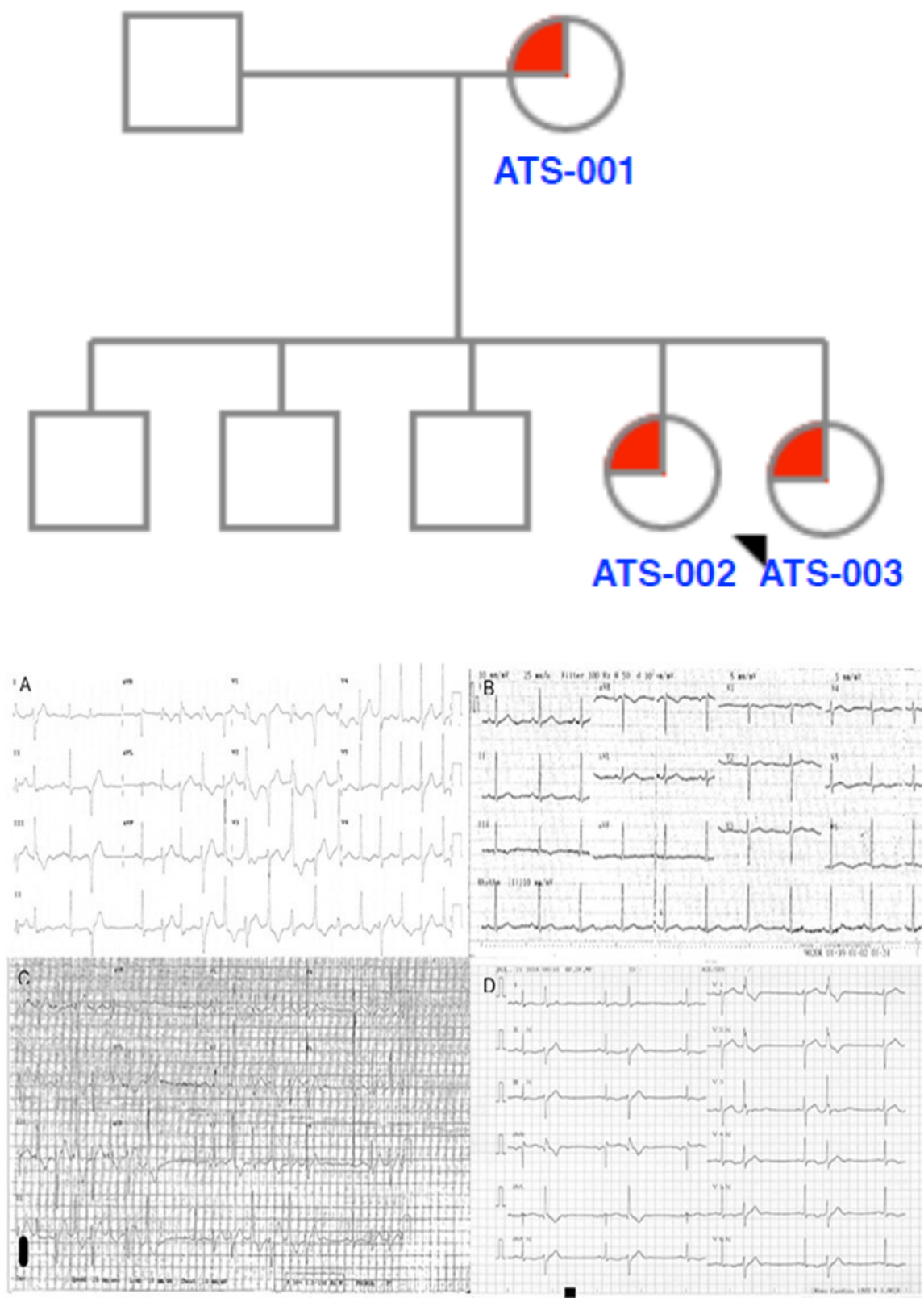

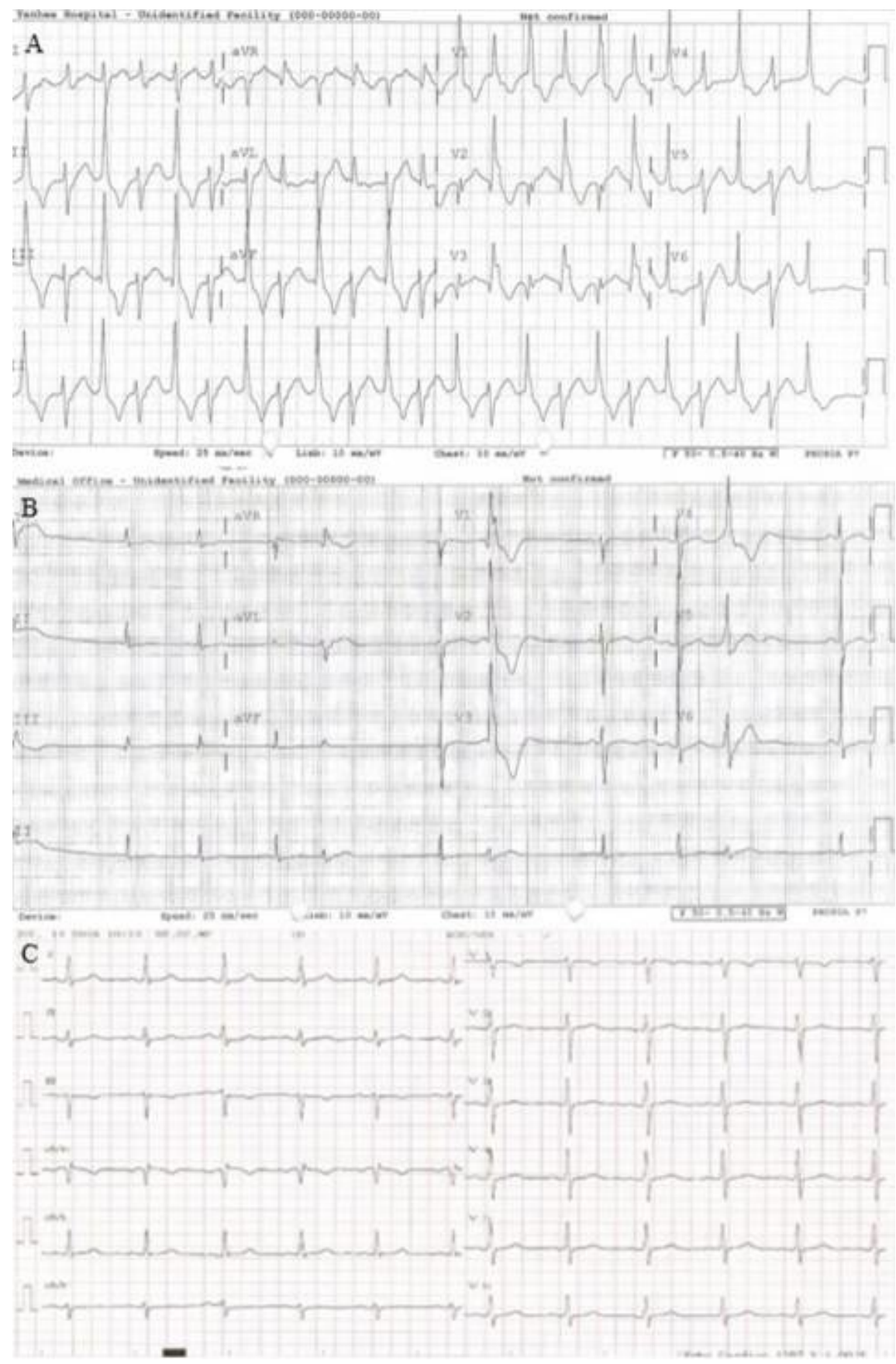

\section{Hosted file}

Table_JCE.rtf available at https://authorea.com/users/331535/articles/458185-successfultreatment-of-arrhythmia-with-\%CE\%B2-blocker-and-flecainide-combination-in-pregnantpatients-with-andersen-tawil-syndrome 